\title{
Krumm, Birgitta
}

\section{Berichte aus den Arbeitskreisen}

Döbrich, Peter [Hrsg.]: Qualitätsentwicklung im naturwissenschaftlichen Unterricht. Fachtagung am 15. Dezember 1999. Frankfurt am Main : GFPF ; DIPF 2002, S. 51-58. - (Materialien zur Bildungsforschung; 7)

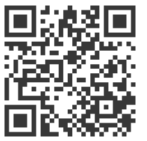

Quellenangabe/ Reference:

Krumm, Birgitta: Berichte aus den Arbeitskreisen - In: Döbrich, Peter [Hrsg.]: Qualitätsentwicklung im naturwissenschaftlichen Unterricht. Fachtagung am 15. Dezember 1999. Frankfurt am Main : GFPF ; DIPF 2002, S. 51-58 - URN: urn:nbn:de:0111-opus-34444 - DOI: 10.25656/01:3444

https://nbn-resolving.org/urn:nbn:de:0111-opus-34444

https://doi.org/10.25656/01:3444

in Kooperation mit / in cooperation with:
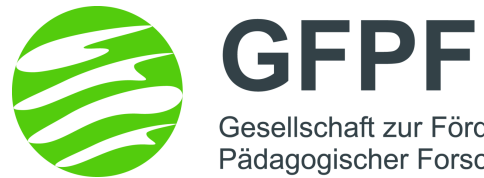

Gesellschaft zur Förderung

Pädagogischer Forschung e.V.

http://www.gfpf.info

\section{Nutzungsbedingungen}

Gewährt wird ein nicht exklusives, nicht übertragbares, persönliches und beschränktes Recht auf Nutzung dieses Dokuments. Dieses Dokument ist ausschließlich für den persönlichen, nicht-kommerziellen Gebrauch bestimmt. Die Nutzung stellt keine Übertragung des Eigentumsrechts an diesem Dokument dar und gilt vorbehaltlich der folgenden Einschränkungen: Auf sämtlichen Kopien dieses Dokuments müssen alle Urheberrechtshinweise und sonstigen Hinweise auf gesetzlichen Schutz beibehalten werden. Sie dürfen dieses Dokument nicht in irgendeiner Weise abändern, noch dürfen Sie dieses Dokument für öffentliche oder kommerzielle Zwecke vervielfältigen, öffentlich ausstellen, aufführen, vertreiben oder anderweitig nutzen.

Mit der Verwendung dieses Dokuments erkennen Sie die Nutzungsbedingungen an.

\section{Terms of use}

We grant a non-exclusive, non-transferable, individual and limited right to using this document.

This document is solely intended for your personal, non-commercial use. Use of this document does not include any transfer of property rights and it is conditional to the following limitations: All of the copies of this documents must retain all copyright information and other information regarding legal protection. You are not allowed to alter this document in any way, to copy it for public or commercial purposes, to exhibit the document in public, to perform, distribute or otherwise use the document in public.

By using this particular document, you accept the above-stated conditions of use.

\section{Kontakt / Contact:}

DeDOCS

DIPF | Leibniz-Institut für Bildungsforschung und Bildungsinformation Informationszentrum (IZ) Bildung

E-Mail: pedocs@dipf.de

Internet: www.pedocs.de

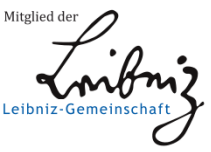


GIDT

Gesellschaft zur Förderung

Pädagogischer Forschung
Deutsches Institut für Internationale

Pädagogische Forschung

Peter Döbrich (Hrsg.)

Qualitätsentwicklung im naturwissenschaftlichen Unterricht

Fachtagung am 15. Dezember 1999

Materialien zur Bildungsforschung

Band 7 
Die Deutsche Bibliothek - CIP-Einheitsaufnahme

Qualitätsentwicklung im naturwissenschaftlichen Unterricht; Fachtagung am 15. Dezember 1999 / GFPF, Gesellschaft zur Förderung Pädagogischer Forschung ; DIPF, Deutsches Institut für Internationale Pädagogische Forschung. Peter Döbrich (Hrsg.) - Frankfurt am Main : GFPF ; Frankfurt am Main : DIPF, 2002

(Materialien zur Bildungsforschung; Bd. 7)

ISBN 3-923638-25-6

Copyright $\odot 2002$ by Gesellschaft zur Förderung Pädagogischer Forschung; Deutsches Institut für Internationale Pädagogische Forschung,

Schloßstraße 29, D-60486 Frankfurt am Main 


\section{Inhalt}

Peter Döbrich; Deutsches Institut für Internationale Pädagogische Forschung, Frankfurt am Main:

Einleitung

Internationale Vergleiche - Rückmeldungen über

die Leistungen unserer Schulen

Wolfgang Gräber, Institut für die Pädagogik der

Naturwissenschaften, Kiel:

„Scientific Literacy“ - Naturwissenschaftliche

Bildung in der Diskussion

Hans Joachim Bezler, Studienrat, Heusenstamm:

Intelligentes Ü̉ben - Behalten - Überprüfen

Positionen einer neuen ,Aufgabenkultur“ im naturwissenschaftlichen Unterricht

Birgitta Krumm, OStD i.R., Frankfurt am Main:

Berichte aus den Arbeitskreisen 
Birgitta Krumm

\section{Berichte aus den Arbeitskreisen}

Arbeitskreis B: Selbständiges und kooperatives Arbeiten - welche Möglichkeiten gibt es und welche Bedeutung kann dies im naturwissenschaftlichen Unterricht haben?

Leitung: Birgitta Krumm, OStD i.R.

"Es gilt, Schüler aus dem Passivum ins Activum zu übersetzen. Nicht übermittelt und übernommen sondern erarbeitet sollen die Unterrichtsstoffe werden, sodass Lernen zum Beruf des Schülers wird" (Hugo Gaudig zitiert in O. Scheibner 1962) oder heute formuliert: "Don't preach facts - stimulate acts".

Folgendes Schema zeigt die ganze Breite der zugehörigen Forderungen: (aus Thillm 23, S. 8)

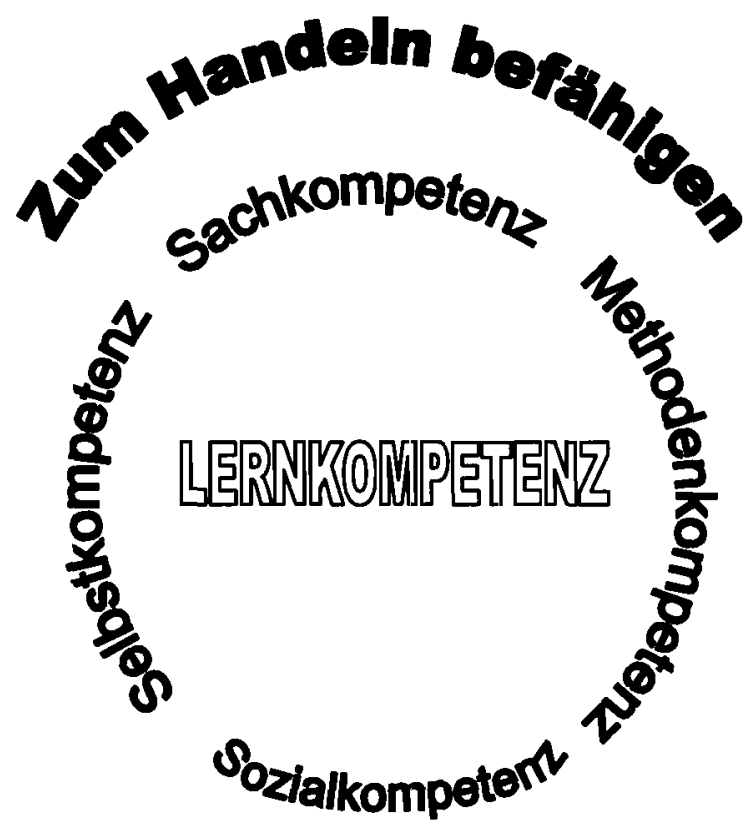


Die Frage, wie man dies vermittelt und erreicht, beschäftigt Didaktiker in allen Bereichen von Hochschule und Schule.

Die Schulleistungsstudien TIMS II und III sowie die angelaufenen Untersuchungen des PISA-Programmes stellen folgende Anforderungen deutlich heraus:

Das Beherrschen von Prozessen

Das Verstehen von Konzepten

Das Anwenden auf wechselnde Situationen

In diesem Arbeitskreis wird deshalb die Frage gestellt, wie man einerseits selbständiges, selbstgesteuertes und andererseits kooperatives Arbeiten im Unterricht erreichen kann; welche Unterrichtsmethoden hierfür geeignet sind und gefordert werden müssen.

Folgendes Schema wurde in der Diskussion entwickelt:

Die Entwicklung einer Lernkultur

\begin{tabular}{|l|l|}
\hline \multicolumn{1}{|c|}{ Selbständig/selbst gesteuert } & \multicolumn{1}{c|}{ kooperativ } \\
\hline $\begin{array}{l}\text { individuelle Lern- und Lösungs- } \\
\text { wege anregen }\end{array}$ & Verständigen üben \\
$\begin{array}{l}\text { erfordert Lern- und Arbeitstech- } \\
\text { niken zur Selbstorganisation }\end{array}$ & $\begin{array}{l}\text { Zusammentragen von Teilfragen } \\
\text { als Bausteine }\end{array}$ \\
$\begin{array}{l}\text { Zeiteinteilung (eigenes Lerntem- } \\
\text { po kennen) }\end{array}$ & $\begin{array}{l}\text { Erfordert geeignete Arrange- } \\
\text { ments ("organisieren können") }\end{array}$ \\
$\begin{array}{l}\text { Fragen verstehen, finden, } \\
\text { Ziel erkennen }\end{array}$ & $\begin{array}{l}\text { Partnerschaftliche Rolle als we- } \\
\text { sentlich erleben }\end{array}$ \\
$\begin{array}{l}\text { Entwicklung und Fortschritt ei- } \\
\text { genen Könnens (Kompetenzzu- } \\
\text { wachs) so oft wie möglich erleb- } \\
\text { bar machen }\end{array}$ & \\
\hline
\end{tabular}


Daraus kann sich Selbstbewusstsein entwickeln.

Die Überlegungen zu den Unterrichtsmethoden lassen sich verschieden strukturieren:

\section{Nach Arbeitsschritten:}

von der Suche nach dem Ziel, dem Erkennen der Thematik bis zur Präsentation

Im Mittelpunkt der Arbeit sollen stehen:

- Methoden des individuellen Arbeitens und

- Methoden der Zusammenarbeit

Zum Beispiel:

Methoden der zielgerichteten Suche, Auswahl und Anwendung von Informationen, Hilfs- und Arbeitsmitteln

Methoden des Ordnens und Speicherns von Informationen

Methoden des Transfers von Informationen

Methoden des Erkennens, Prüfens, Lösens von Problemen und der Überprüfung der Lösungen

Methoden der Reflexion eigener und fremder Arbeit

Methoden der Präsentation, Verteidigung und Anwendung von Arbeitsergebnissen.

Die Auswahl der anzuwendenden Methoden sollte exemplarischen Charakter haben.

2. Die unterrichtsmethodischen Verfahren lassen sich nach Verwendung beim Start, beim Lösungsprozess und bei der Ergebnisfeststellung sichten. Die Frage, welches Verfahren sich für unser Ziel bei unseren Voraussetzungen und Möglichkeiten am besten eignet, muss die Auswahl und Anwendung leiten.

Für den Beginn:

Brainstorming

Mindmapping

Clustering

Texte / Reklame

Sprech-, Denkblasen schreiben

sächliche Motivationen u.ä. 
Für den Lösungsprozess:

Gruppen-, Partner-, Einzelarbeit

Lernen an Stationen

Gruppenpuzzle

Wochenplan, Projektarbeit

Expertenbefragung, Betriebsbesichtigung

Tagebuch, Logbuch, Portfolios

Planspiel, Rollenspiel

Neue Medien: geeignete Software, Internetnutzung

Für die Ergebnisdarstellung:

Präsentationsformen: Referate, Ausstellung, Modelle, Szenen.

Rollen- und Planspiel

Podiumsdiskussion

3. Hier sollen noch andere Überlegungen ins Blickfeld genommen werden, um die Forderungen des PISA-Projektes bewusst zu machen.

Das Beherrschen von Prozessen bedeutet

a) an Problemen lernen

Das Verstehen von Konzepten gelingt nur bei

b) Anknüpfen an die Vorerfahrungen von Schülern und Schülerinnen und

c) Erreichen von Vorstellungswechsel (Conceptual Change)

Das Anwenden auf wechselnde Situationen erfordert

d) das Entwickeln von Sozial- und Ichkompetenz

e) das Wecken von langfristigem Interesse

f) fachübergreifendes und fächerverbindendes Denken in verschiedenen Zusammenhängen (Cross-Curricular-Competencies).

$Z u 3 a):$

Zentrale Aufgabe des naturwissenschaftlichen Unterrichts ist das problemorientierte Lernen. Natürlich lässt sich eine Unterrichtsstunde oder kürzere wie längere Phasen als "forschender" oder "problemlösender" 
Unterricht auch durch entsprechend vom Lehrer geleiteten fragendentwickelnden Ablauf demonstrieren. Die Frage ist jedoch, ob instruktiv geführter Unterricht zur ausreichenden Schulung des Einzelnen führt, ob dadurch Problemlösestrategien tatsächlich erkannt und außerhalb, nach der Schulzeit in dieser Weise selbstverständlich gearbeitet wird, ob so Lernen stattfindet.

Nach heutiger Erkenntnis muss Problemorientierung zumindest eine Balance zwischen Instruktion und Konstruktion fordern. Die Einbettung in authentische Kontexte (Glassammeln, Kunststoffflasche, Auge, Brille ...) und soziale Kontexte (Glasrecycling, Fotogeschäft, Augenoptiker, Augenarzt) wie auch in multiple Kontexte (in verschiedene Situationen einbetten: Glas in der Schule, verschiedene Brillen in der Familie, Glasfaser in der Kommunikationstechnik ...) sind ebenso notwendig wie Instruktionen (diese können vom Lehrer aufbereitet sein oder aus den notwendigen Medien vom Buch bis Internet stammen).

\section{$Z u 3 b)$ und c):}

Seit vielen Jahren vorhandene Beobachtungen im naturwissenschaftlichen Unterricht belegen, dass die Vorerfahrungen aus frühestem Jugendalter für den naturwissenschaftlichen Unterricht in der Sekundarstufe I als nicht unbedingt hilfreich empfunden werden. Missverständnisse zwischen Schülervorstellungen und den Erkenntnissen der Wissenschaft werden meistens nicht aufgedeckt und führen dazu, dass das Gebäude der Alltagsvorstellungen nach der Schulzeit allein stehen bleibt und das erworbene Wissen verschwindet.

Ähnliches gilt für das sprachliche Weltbild, die Alltags- und Fachsprache. In einem Unterricht, der nicht nur auf reinen Wissenserwerb zielt, der den Prozess dahin als wesentlichen Bestandteil ansieht, muss die Lehrkraft Alltagsbegriffe kennen, die zum Teil üblich, zum Teil mit anderem Sinn gelten, und diese antizipieren, auf sie hören: hinhören, Antworten fixieren, Schülerdiskussionen zur Begriffserklärung zulassen bzw. initiieren.

Hilfreich sind hier sicher sogenannte Interaktionsanalysen und fördernd die Portfoliokultur. 
Zu 3 d):

Hier wird die Notwendigkeit besonders klar Methoden anzuwenden, die kooperatives Lernen fördern und andererseits solche, die Selbststeuerung erfahrbar machen und somit beides schulen.

Hierher gehören verschiedene Gruppenbildungsvarianten (Freundesgruppen, oder der Zufall oder der Lehrer entscheidet).

Vorangehen kann die Themenfindung für die Arbeitsgruppen oder für die Einzelarbeit. Es geht darum, Regeln zu finden und festzulegen, wie gearbeitet wird.

Die Gruppenmitglieder üben den Umgang mit Literatur (Literatur zu Glas sichten, in Teilthemen aufgliedern), sie kümmern sich um Möglichkeiten zur praktischen Arbeit, zum Experimentieren (Glasblasen, Glasherstellung, Glaseigenschaften), sie suchen den täglichen Bezug (Glasmüll in der Schule, zu Hause, Pfandflaschen).

Zu $3 e)$ :

Wie erreicht man ein langfristiges Interesse?

Dies soll mit folgenden Hinweisen, die offenbar wirken können, umrissen werden:

Jugendliche müssen die Erfahrung machen, dass die Themen mit ihnen zu tun haben.

Sie sollen spüren, dass sie und ihre Wahrnehmungen oder Gedanken ernst genommen werden.

Das Umgehen mit den eigenen Kenntnisdefiziten beim Gespräch mit Experten soll geübt werden.

Der Unterricht darf nicht so geleitet werden, dass der Lehrer jeweils die Spreu vom Weizen trennt, jedoch sind Strukturierungshilfen und vielfältige Übungsmöglichkeiten zum Verhindern von Enttäuschung oder Frust notwendig.

Das Schaffen des Arbeitserlebnisses für den Jugendlichen, das ein Bewusstsein des Eigentums (Scheibner) hervorruft, sollte immer bedacht werden.

Das Einbeziehen der Eltern, des Schülerumfeldes verknüpft Schule und Herkunft. 
Es wird deutlich, dass hier Gruppen- und Einzelarbeit, das Erfragen und Erkunden, das Erstellen von Produkten aller Art, das Rollenspiel und szenisches Darstellen brauchbare Methoden sein können.

$\mathrm{Zu} 3 \mathrm{f})$ :

Schüler und Schülerinnen müssen zusammen mit der Lehrkraft Fragen an die anderen Fächer richten und diese nicht nur auf Fragen antworten. Dies Vorgehen erfordert wohl die größte Methodenvielfalt. Wichtig ist eine übersichtliche Planung und Zuordnung wie auch die inhaltliche und methodische Absprache mit den anderen Unterrichtenden. Am Beispiel Glas soll dies wiederum dargestellt werden (s. Anhang).

\section{Schlussbetrachtung:}

In der Diskussion mit den zwei Teilnehmergruppen wurde besonders deutlich, dass lehrerdominierte Unterrichtsformen überwiegen, vor allem ein fragend-entwickelndes Unterrichtsgespräch, das auf eine Lösung zielt, die der Lehrer im Kopf hat. Schülerexperimente werden als dringend notwendig angesehen, scheitern jedoch an den Lerngruppengrößen.

Variable Lernformen, z.B. Lernen an Stationen scheitern offenbar auch an räumlichen Möglichkeiten (,wo lässt man die Stationen über eine Woche stehen, wann ist in der Schule Zeit vorhanden, etwas vorzubereiten, aufzubauen?"“). Gruppenteiliges Arbeiten wird in den oft zu kleinen Unterrichtsräumen als fast unmöglich zu realisieren dargestellt, fächerverbindendes Arbeiten im Kollegium mangels Koordinierungsstunden bzw. möglichkeiten kaum verwirklicht. Die Erfüllung des Lehrplanes, Probleme beim Führen und Begleiten von Schüler-Schülergesprächen und die Schwierigkeiten; einen ,roten Faden" zu halten sowie Erreichtes als Verpflichtung zu fixieren, sind weitere Belastungen bei den Versuchen, Schule im Sinne des Geforderten zu verändern. 


\section{SOZIALKUNDE}

Soziale Situation im Mittelgebirge im MA

\section{GESCHICHTE}

Fenster

Geschichte der

Glashütten

\section{CHEMIE}

Rohstoffe, Herstellung, Recycling

Eigenschaften

\section{GLAS}

\section{INFORMATIK}

Rolle des Chip

\section{Auge Linse \\ BIOLOGIE}

Mikroskopie: Blick in die Mikrowelt 